\title{
Modelling of Boltzmann transport equation for freeze-out
}

\author{
K Tamosiunas ${ }^{1,2}$, L P Csernai ${ }^{1,3}, \mathbf{V}$ K Magas ${ }^{4}, \mathbf{E}$ Molnár $^{1}$ and Á Nyíri ${ }^{1}$ \\ ${ }^{1}$ Section for Theoretical and Computational Physics and Bergen Computational Physics \\ Laboratory, BCCS-Unifob, University of Bergen, Allegaten 55, 5007 Bergen, Norway \\ ${ }_{2}^{2}$ Department of Physics, Vytautas Magnus University, Donelaicio 58, 3000 Kaunas, Lithuania \\ ${ }^{3}$ MTA-KFKI, Research Institute of Particle and Nuclear Physics, H-1525 Budapest 114, \\ PO Box 49, Hungary \\ ${ }^{4}$ Departamento de Física Teórica and IFIC Centro Mixto, Universidad de Valencia-CSIC, \\ Institutos de Investigación de Paterna Apdo. correos 22085, 46071, Valencia, Spain \\ E-mail: Karolis.Tamosiunas@ift.uib.no
}

Received 20 November 2004

Published 23 May 2005

Online at stacks.iop.org/JPhysG/31/S1001

\begin{abstract}
The freeze-out (FO) in high-energy heavy-ion collisions is assumed to be continuous across finite layer in space-time. Particles leaving local thermal equilibrium start to freeze out gradually till they leave the layer, where all the particles are frozen out. To describe such a kinetic process we start from Boltzmann transport equation (BTE). However, we will show that the basic assumptions of BTE, such as molecular chaos or spatial homogeneity do not hold for the above-mentioned FO process. The aim of the presented work is to analyse the situation, discuss the modification of BTE and point out the physical causes, which yield to these modifications of BTE for describing FO.
\end{abstract}

\section{Continuous freeze-out and Boltzmann transport equation}

For introduction let us describe the continuous freeze-out (FO) process in the layer. FO is an important phase in dynamical processes and is mostly assumed to be discontinuous in space-time (ST) and described by three-dimensional hypersurface, separating local thermal equilibrium phase and fully frozen, i.e. non-interacting matter. In order to have more realistic description of the FO let us take two closed and disjoint three-dimensional hypersurfaces $S_{1}$ and $S_{2}$ in ST. At early times, centrally in the collision region we have intensively interacting matter, which is equilibrated and thermalized; this is the fluid-dynamical domain bordered by the $S_{2}$ hypersurface, which has a normal 4-vector $\mathrm{d} \sigma_{\mu}$ and can be space-like or time-like [1]. The location of this surface is given by the fact that the interacting fluid is cooling and expanding, and reaches a point when interactions are not frequent enough to maintain full thermal and hydrodynamical equilibrium locally. Some particles will not interact any 
more beyond this hypersurface. Later on in the expansion and cooling we reach an other hypersurface, $S_{1}$. By reaching surface $S_{1}$ on their way, all particles become non-interacting or free. Thus, when reaching this surface the FO process is completed. The phase-space distribution of particles does not change any more. Between $S_{2}$ and $S_{1}$ we have a layer, where frozen particles are produced. To describe such a far from equilibrium process one can use Boltzmann transport equation (BTE), which may describe equilibrium and non-equilibrium processes equally well.

The BTE can be derived from the conservation of charges in a ST domain, $\Delta^{4} x$, assuming the standard conditions [2]: (i) only binary collisions are considered, (ii) 'molecular chaos' assumption, i.e. that the number of binary collisions at position $x$ is proportional to $f\left(x, p_{1}\right) \times f\left(x, p_{2}\right)$ and that (iii) $f(x, p)$ is a smoothly varying function compared to the mean free path.

For the PS integration over the momenta of the other three particles participating in the binary collision we use the notation: ${ }_{12} \mathcal{D}_{3} \equiv \frac{\mathrm{d}^{3} p_{1}}{p_{1}^{0}} \frac{\mathrm{d}^{3} p_{2}}{p_{2}^{0}} \frac{\mathrm{d}^{3} p_{3}}{p_{3}^{0}}$. Shortening the notation further, by dropping the momentum arguments of the PS distributions and keeping the indices only, BTE reads

$$
p^{\mu} \partial_{\mu} f=\frac{1}{2} \int{ }_{12} \mathcal{D}_{4} f_{1} f_{2} W_{12}^{p 4}-\frac{1}{2} \int{ }_{2} \mathcal{D}_{34} f f_{2} W_{p 2}^{34} .
$$

Now, aiming for the description of freeze-out, let us split up the distribution function, $f$, into two distribution functions: $f=f^{\mathrm{i}}+f^{\mathrm{f}}$, where $f^{\mathrm{f}}$ is the phase-space distribution function of the 'free' or frozen-out particles, which do not collide any more, while $f^{\mathrm{i}}$ is the interacting component $[3,4]$. Thus, the freeze-out process is represented here by gradually populating and building up the 'free' component while draining particles from the interacting component. As the particles belonging to the free component may not collide any more, they do not appear in the initial state components of collision integrals! The gain term, $f_{1} f_{2} W_{12}^{p 4} \equiv f_{1}^{\mathrm{i}} f_{2}^{\mathrm{i}} W_{12}^{p 4}$, populates both the interacting, $f^{\mathrm{i}}$, and free, $f^{\mathrm{f}}$, components, so we will introduce a FO probability, $\mathcal{P}_{\mathrm{f}}$, which 'feeds' the free component. The probability is phase-space dependent, but it may depend on the positions and momenta of both incoming particles, and it can weight the outgoing phase space for one (or both) outgoing particles. In the most simple case we have to assume that it depends at least on the momentum of the outgoing particle, which belongs to the component $f^{\mathrm{f}}: \mathcal{P}^{\mathrm{FO}}(x, p) \equiv \mathcal{P}_{\mathrm{f}}$. Thus, the complete BTE yields

$p^{\mu} \partial_{\mu}\left(f^{\mathrm{i}}+f^{\mathrm{f}}\right)=\frac{1}{2} \int{ }_{12} \mathcal{D}_{4} f_{1}^{\mathrm{i}} f_{2}^{\mathrm{i}}\left[\mathcal{P}_{\mathrm{f}} W_{12}^{p 4}+\left(1-\mathcal{P}_{\mathrm{f}}\right) W_{12}^{p 4}\right]-\frac{1}{2} \int{ }_{2} \mathcal{D}_{34} f^{\mathrm{i}} f_{2}^{\mathrm{i}} W_{p 2}^{34}$

We can separate above equation into two equations. Sum of them returns BTE:

$$
\begin{aligned}
& p^{\mu} \partial_{\mu} f^{f}=\frac{1}{2} \int{ }_{12} \mathcal{D}_{4} f_{1}^{\mathrm{i}} f_{2}^{\mathrm{i}} \mathcal{P}_{\mathrm{f}} W_{12}^{p 4} \\
& p^{\mu} \partial_{\mu} f^{\mathrm{i}}=-\frac{1}{2} \int{ }_{12} \mathcal{D}_{4} f_{1}^{\mathrm{i}} f_{2}^{\mathrm{i}} \mathcal{P}_{\mathrm{f}} W_{12}^{p 4}+\frac{1}{2} \int{ }_{12} \mathcal{D}_{4} f_{1}^{\mathrm{i}} f_{2}^{\mathrm{i}} W_{12}^{p 4}-\frac{1}{2} \int{ }_{2} \mathcal{D}_{34} f^{\mathrm{i}} f_{2}^{\mathrm{i}} W_{p 2}^{34} .
\end{aligned}
$$

The free component does not have a loss term because particles in the free component do not interact anymore and cannot be lost. The first term of the second equation is a drain term, describing the escape or FO of particles from the interacting component. It is the inverse of the gain term for the free component. The last two terms are influencing the interacting component, and do not include the FO probability factors! Thus, these two terms drive the interacting component towards re-thermalization. For these the relaxation time approximation can be used [5, 7]: 


$$
p^{\mu} \partial_{\mu} f^{\mathrm{i}}=-\frac{1}{2} \int{ }_{12} \mathcal{D}_{4} f_{1}^{\mathrm{i}} f_{2}^{\mathrm{i}} \mathcal{P}_{\mathrm{f}} W_{12}^{p 4}+p^{0} \frac{f_{\text {eq }}^{\mathrm{i}}-f^{\mathrm{i}}}{\tau_{\text {rel }}} .
$$

\section{Modified Boltzmann transport equation}

Now, the question arises, can the BTE handle realistically the FO process? We have seen that the structure of the kinetic equations, used earlier to describe freeze-out [4-7], and the separation of the 'escape' and 're-thermalization' terms come out in a simple, straightforward way from the BTE.

The usual structure of the collision terms in the BTE is not adequate for describing rapid FO in a layer, which is comparable to the mean free path. If we assume the existence of such a layer (where density gradient in the $\mathrm{d} \sigma_{\mu}$ direction is very high), this immediately contradicts assumption of BTE (see point (iii)): the change is not negligible in the direction of $\mathrm{d} \sigma^{\mu}$. The assumption of 'molecular chaos' is also violated in a FO process because the density of interacting particles is much higher near hypersurface $S_{2}$ and goes to zero on the hypersurface $S_{1}$. Thus, number of collisions is not proportional with $f\left(x, p_{1}\right) \times f\left(x, p_{2}\right)$, but it is delocalized in the normal direction, $\mathrm{d} \sigma_{\mu}$, with $f\left(x_{1}, p_{1}\right) \times f\left(x_{2}, p_{2}\right)$, where $x_{k}$ is the origin of colliding particles, i.e. the ST point where the colliding particles were colliding last, $x_{k}=x-u_{k} \tau_{k}, \tau_{k}$ is the collision time, $u_{k}^{\mu}=\left(\gamma_{k}, \gamma_{k} \vec{v}_{k}\right), \gamma=1 / \sqrt{1-\vec{v}^{2}}$ and $\vec{v}_{k}=\vec{p}_{k} / p_{k}^{0}$. The appropriate equations to describe continuous FO can be a modified Boltzmann transport equation (MBTE), following equations (3) and (5):

$$
\begin{aligned}
& p^{\mu} \partial_{\mu} f^{\mathrm{f}}(x, p)=\frac{1}{2} \int{ }_{12} \mathcal{D}_{4} \mathcal{P}_{\mathrm{f}} W_{12}^{p 4} f^{\mathrm{i}}\left(x_{1}, p_{1}\right) f^{\mathrm{i}}\left(x_{2}, p_{2}\right), \\
& p^{\mu} \partial_{\mu} f^{\mathrm{i}}(x, p)=-\frac{1}{2} \int{ }_{12} \mathcal{D}_{4} \mathcal{P}_{\mathrm{f}} W_{12}^{p 4} f^{\mathrm{i}}\left(x_{1}, p_{1}\right) f^{\mathrm{i}}\left(x_{2}, p_{2}\right)+p^{0} \frac{f_{\mathrm{eq}}^{\mathrm{i}}-f^{\mathrm{i}}}{\tau_{\text {rel }}} .
\end{aligned}
$$

This is an essential modification if the PS distribution has a large gradient in the spacetime. This gradient defines a ST 4-vector, characterizing the direction of the process, $\mathrm{d} \sigma^{\mu}$.

A simple general solution of the MBTE (6), (7) cannot be given, but it serves as a basis for simplified, phenomenological kinetic models describing the freeze-out process.

\section{Lorentz-Boltzmann linear approximation and escape probability}

The starting point to solve MBTE may be Lorentz-Boltzmann linear approximation, where deviation from locally equilibrated distribution, $f_{k}^{(0)}\left(x, p_{k}\right)$, is taken into account as a small correction, $\phi\left(x_{k}, p_{k}\right)$, around $x[8]$ :

$$
f_{k}\left(x_{k}, p_{k}\right)=f_{k}^{(0)}\left(x, p_{k}\right)\left[1+\phi\left(x_{k}, p_{k}\right)\right]
$$

where $\phi\left(x_{k}, p_{k}\right)$ is delocalized function and it keeps the norm of the distribution function $f_{k}\left(x_{k}, p_{k}\right)$. The deviation, $\phi\left(x_{k}, p_{k}\right)$, from equilibrated distribution is due to (i) on the interacting side, momentum distribution is more spread out than on the FO side, because of the cooling; (ii) the density of interacting particles decreasing going towards FO side (in the direction of $\left.\mathrm{d} \sigma_{\mu}\right) . \phi\left(x_{k}, p_{k}\right)$ can be approximated as $\phi_{k}\left(x_{k}, p_{k}\right) \approx \phi_{k}\left(x, p_{k}\right)+$ $\left(x_{k}^{\mu}-x^{\mu}\right) \partial_{\mu} \phi_{k}\left(x, p_{k}\right)+c_{p \phi} p_{k}^{\mu} \mathrm{d} \sigma_{\mu}$. Particles with momentum $p_{k}^{\mu}$ propagates from $x_{k}^{\mu}$ to $x^{\mu}$, so that $\left(x_{k}^{\mu}-x^{\mu}\right)=-c_{x \phi} p_{k}^{\mu}$, then $\phi_{k}\left(x_{k}, p_{k}\right) \approx \phi_{k}\left(x, p_{k}\right)+c_{\phi} p_{k}^{\mu} \mathrm{d} \sigma_{\mu}$.

Now using above approximation and inserting (8) into the MBTE (6), we get

$$
p^{\mu} \partial_{\mu} f^{\mathrm{f}}(x, p)=\frac{1}{2} f^{(0)}(x, p) \int{ }_{12} \mathcal{D}_{3} f_{3}^{(0)} \mathcal{P}_{\mathrm{f}} W_{12}^{3 p}\left(\phi_{1}+\phi_{2}\right),
$$

where $f^{(0)}(x, p)$ is locally equilibrated interacting distribution and $f_{3}^{(0)}$ is distribution of the 
particle leaving the collision. Such a form of MBTE is more transparent, because just the $\phi_{1}=\phi\left(x_{1}, p_{1}\right)$ and $\phi_{2}=\phi\left(x_{2}, p_{2}\right)$ are influenced of ST configuration of the FO layer. Performing the integral over $p_{3}$ leads to an averaging over transition rate. And assuming small angle scatterings, that the transition rate $W_{12}^{3 p} \approx \omega \delta\left(p-p_{1}\right) \delta\left(p-p_{2}\right)$, we can rewrite equation (9) as

$$
p^{\mu} \partial_{\mu} f^{\mathrm{f}}(x, p)=f^{(0)}(x, p) n^{\mathrm{i}}(x) \mathcal{P}_{\mathrm{f}} \frac{\left(p^{\mu} \mathrm{d} \sigma_{\mu}\right)^{2}}{p^{0}},
$$

where $n^{\mathrm{i}}(x)=\int \frac{\mathrm{d}^{3} p_{3}}{p_{3}^{0}} f_{3}^{(0)}\left(x, p_{3}\right)$.

In order to solve the continuous emission of particles in a layer, we introduce escape probability: $\mathrm{d} \sigma^{\mu} \partial_{\mu} f^{\mathrm{f}}(x, p)=f^{\mathrm{i}}(x, p) P_{\mathrm{esc}}^{*}$. It depends on the ST coordinates, on the interacting part of the PS distribution, on the transfer properties and FO probability: $P_{\text {esc }}^{*}\left(x, p, f^{\mathrm{i}}, \mathrm{d} \sigma, w, \mathcal{P}_{\mathrm{f}}\right)$. The derivation above did neglect several details and features, however, reflects the basic structure of ad hoc kinetic FO models [4-7]. The invariant escape probability, within the FO layer (length of a layer denoted by $L$ ), covering both the time-like and space-like parts of the layer, $P_{\mathrm{esc}}^{*}=\frac{1}{\lambda\left(\bar{x}_{1}\right)}\left(\frac{L}{L-x^{\mu} \mathrm{d} \sigma_{\mu}}\right)^{a}\left(\frac{p^{\mu} \mathrm{d} \sigma_{\mu}}{p^{\mu} u_{\mu}}\right)^{a} \Theta\left(p^{\mu} \mathrm{d} \sigma_{\mu}\right)$. The more detailed investigation about escape probability $P_{\mathrm{esc}}^{*}(p)$ for different $\mathrm{d} \sigma_{\mu}$ can be found in $[9,10]$.

\section{Conclusions}

The freeze-out process was discussed in the four-dimensional space-time in a layer of finite thickness. Arising from the physical process this layer is directed, it has an inside and outside boundary, which are not identical. The processes in the layer are not isotropic, they must be sensitive to the direction of the layer. It is shown that, as a consequence, the basic assumptions of the Boltzmann transport equation are not satisfied in this layer, and the equation should be modified. It is also shown that earlier ad hoc kinetic models of the freeze-out process can be obtained from this approach in a fully covariant way, and freeze-out in space-like and time-like directions can be handled on the same covariant footing.

\section{Acknowledgments}

The authors wish to thank J Cleymans and his colleagues for their hospitality at the University of Cape Town, where parts of this work were done in the framework of a Norwegian-South African collaborative research project.

\section{References}

[1] Csernai L P 1987 Zh. Eksp. Teor. Fiz. 92379 Csernai L P 1987 Sov. JETP 65 (2)

[2] Csernai L P 1994 Introduction to Relativistic Heavy Ion Collisions (New York: Wiley)

[3] Sinyukov Yu M, Akkelin S V and Hama Y 2002 Phys. Rev. Lett. 89052301 Akkelin S V, Borysova M S and Sinyukov Yu M 2004 Preprint nucl-th/0403079

[4] Csernai L P, Lázár Zs and Molnár D 1997 Acta Phys. Hung. New Ser. Heavy Ion Phys. 5467

[5] Magas V K et al 1999 Acta Phys. Hung. New Ser. Heavy Ion Phys. 9193

[6] Magas V K et al 1999 Nucl. Phys. A 661596 Anderlik Cs et al 1999 Phys. Lett. B 45933

[7] Magas V K, Anderlik A, Anderlik Cs and Csernai L P 2003 Eur. Phys. J. C 30 255-61

[8] De Groot S R 1980 Relativistic Kinetic Theory: Principles and Applications (Amsterdam: Elsevier)

[9] Csernai L P et al 2004 Preprint hep-ph/0401005

[10] Csernai L P et al 2004 Preprint hep-ph/0406082 